\title{
Direccionalidad de los estilos parentales en la regulación emocional infantil ${ }^{1}$ \\ Directionality of parental styles in children's emotional regulation
}

pp. $76-88$

Diana Sofia Fernandez Erazo ${ }^{2}$

REC: $31 / 03 / 2021$

Hellen Geraldin Ortiz ZÚÑ IGa 3

ACEP: $8 / 07 / 2021$

JORGE ENRIQUE QUIMBAYA GOMEZ ${ }^{4}$

\section{Resumen}

El presente artículo responde a una revisión bibliográfica de tipo cualitativo, el cual tiene como objetivo la comprensión teórica y conceptual de la direccionalidad de los estilos parentales (EP) y su relación con la regulación emocional infantil. Es importante reconocer que los EP resultan fundamentales en el aprendizaje y desarrollo de la regulación emocional (RE) dado que, dependiendo de la forma de interacción, normatización y afecto parental que los niños experimenten en sus hogares por parte de sus cuidadores primarios, pueden influir en cómo los niños logren reconocer sus emociones para luego tener un control sobre ellas y responder de manera óptima frente a situaciones específicas que se les presenten. Lo anterior contribuye a la comprensión del desarrollo integral de los niños, propicia la generación de espacios de formación en competencias emocionales, como también el desarrollo de estrategias y habilidades de afrontamiento que posibilitan el manejo de las emociones y la adaptación del sujeto en su entorno.

Palabras clave: estilos parentales, regulación emocional, desarrollo infantil.

1. Producto del anteproyecto del trabajo de grado titulado "Direccionalidad de los estilos parentales y su incidencia en la regulación emocional: una revisión teórico conceptual desde el enfoque cognitivo conductual".

2. Estudiante de Psicología, noveno semestre, de la Fundacion Universitaria Católica Lumen Gentium. Correo electrónico: sofia9714fer@gmail.com-Orcid: https:// orcid.org/0000-0002-0782-7866

3. Estudiante de Psicología, noveno semestre, de la Fundacion Universitaria Católica Lumen Gentium. Correo electrónico: hegeorzu24@gmail.com-Orcid: https:// orcid.org/0000-0003-0011-2822

4. Magíster en Psicología de la Universidad de San Buenaventura Cali. Docente, Fundación Universitaria Católica Lumen Gentium. Docente orientador del Semillero de Investigación en Evaluación Psicológica y Desarrollo Humano - SINEPSI. Correo electrónico: jequimbaya@unicatolica.edu.co - Orcid: https:// orcid.org/0000-0003-1456-9874 


\section{Abstract}

This article responds to a qualitative bibliographic review, which aims at the theoretical and conceptual understanding of the directionality of parental styles (PS) and its relationship with infantile emotional regulation. It is important to recognize that PSs are fundamental in the learning and development of emotional regulation (ER) since, depending on the form of interaction, normatization and parental affection that children experience at home by their primary caregivers, they can influence how children manage to recognize their emotions to later have control over them and respond optimally to specific situations that arise. The above contributes to the understanding of the integral development of children, fosters the generation of training spaces in emotional competencies, as well as the development of coping strategies and skills that enable the management of emotions and the adaptation of the subject in their environment.

Keywords: parenting styles, emotion regulation, development.

\section{Introducción}

En los entornos familiares son diversos los estilos parentales (EP) que se desarrollan con el fin de establecer la crianza, el cuidado y la educación de los niños. Ante ello, cada familia dentro de su dinámica tiene una forma de funcionar, esto va a determinar el EP que utilizarán en el proceso de normatización en la crianza de los niños. El EP es la forma como los padres o cuidadores ejercen el establecimiento de normas y el manejo de autoridad con los niños (Baumrind, 1967; Izzedin y Pachajoa, 2009; Solís y Díaz, 2008). Es a partir de esto que Baumrind (1967) categoriza las formas de funcionamiento parentales en: EP permisivo, autoritario y democrático.

Los EP se identifican de acuerdo con la manera en que los padres o cuidadores se relacionan e introducen normas frente a la educación de los niños (Tabla 1). Los EP pueden influir adecuada o inadecuadamente en la manera como los niños aprenden a reconocer, expresar y regular sus emociones para adoptar comportamientos y acciones adecuadas al enfrentarse a situaciones específicas, lo cual permitirá la adaptación del sujeto en su entorno (Baumrind, 1975; Darling y Steinberg, 1993). Es importante tener presente que los EP contribuyen al desarrollo integral de los niños, dado que propician la formación de competencias emocionales, así como también el desarrollo de estrategias y habilidades de afrontamiento que posibilitan el manejo de las emociones (Maccoby, 1983; Baumrind, 1967).

Tabla 1

Descripción de los EP.

\begin{tabular}{cl}
\hline $\begin{array}{c}\text { Estilo } \\
\text { parental }\end{array}$ & \multicolumn{1}{c}{ Características } \\
\hline Autoritario & $\begin{array}{l}\text { Alto grado de exigencias (control), } \\
\text { castigos irracionales, baja } \\
\text { comunicación, y deficiente afecto }\end{array}$ \\
\hline Permisivo & $\begin{array}{l}\text { Bajo control, no hay instauración de } \\
\text { normas y con muestra de afectividad } \\
\text { hacia sus hijos }\end{array}$ \\
\hline Democrático & $\begin{array}{l}\text { Instauración de normas y control } \\
\text { racional unido con afectividad, } \\
\text { comprensión y comunicación. }\end{array}$ \\
\hline
\end{tabular}

Fuente: Elaboración propia.

Teniendo en cuenta que las formas de interacción familiar se establecen para llevar a 
cabo el proceso de normatización y crianza de los niños, es indispensable revisar ante qué situaciones se convierten en un promotor de problemáticas sociales. En Colombia, diversas investigaciones apuntan al hecho de que el EP autoritario es negativo, puesto que ejerce una autoridad inhibidora; esto genera alteraciones de tipo emocional y comportamental que intervienen directamente en la salud mental de los niños, lo cual da origen a problemáticas de tipo relacional, comportamental y emocional debido a que el niño no cuenta con competencias para adaptarse fácilmente a su entorno (Moreno et al., 2020; García y Restrepo, 2017; Montoya et al., 2016; Aldana y Siena, 2018; Varela et al., 2015).

Sin embargo, los EP que ejercen una normatización racional, en la cual tienen presente el afecto parental al momento de imponer normas, propician en los niños la interiorización de respuestas más adaptativas, las cuales les permiten una regulación emocional (RE) para responder a situaciones específicas de su contexto social (Baumrind, 1966; Suárez y Vélez, 2018; Aldana y Siena, 2018; Varela et al., 2015).

Ante este panorama se observa que los estudios acerca de las influencias de los EP en el desarrollo infantil se abordan desde una sola dirección, es decir, se hace énfasis en el rol de los padres o cuidadores, pero se pierden de vista las acciones de otros actores presentes en la crianza, así como el lugar del niño en estas interacciones.

De acuerdo con lo anterior, la presente revisión documental tiene como objetivos, en primera instancia, reconocer los diferentes aportes teóricos y conceptuales que se han realizado al estudio de los EP y la RE; analizar la dirección de las relaciones en los EP y cómo la unidireccionalidad 0 bidireccionalidad propician un papel activo o pasivo a cada uno de los actores involucrados en la dinámica de la crianza. En efecto, se espera de esta manera avanzar en la comprensión de diferentes problemáticas sociales en el ámbito del desarrollo infantil, tales como: desórdenes emocionales, psicopatologías, problemas para el desarrollo de las habilidades sociales y conducta prosocial; también, alteraciones en el comportamiento que conllevan a conductas disruptivas y agresivas debido a modelos de crianza irracionales (Moreno et al., 2020; Cuervo, 2010).

\section{M etodología}

Para el presente artículo se tuvo en cuenta el método de la revisión bibliográfica de tipo cualitativo, que permitió la investigación documental de distintas fuentes de información primarias y secundarias como artículos, revistas y documentos, para dar cumplimiento al objetivo de la revisión documental que es identificar la direccionalidad de los EP y su incidencia en la RE infantil. Para lo anterior, la recopilación de la información se hizo por medio de buscadores web de carácter académico como Dialnet, SciELO, Redalyc, APA PsycNet, Google Académico, PDF Drive, Repositorio Ruidera-UCLM y Repositorio PUCP. Dentro de la revisión de bibliografía, se recopilaron 50 artículos científicos de los cuales, a partir de un análisis del contenido de la información, se creó una categoría de análisis que facilitara la comprensión del fenómeno.

Por otro lado, se definieron los siguientes criterios de inclusión: para la búsqueda de documentos se tuvo en cuenta palabras clave como estilos parentales y regulación emocional; en la recolección de documentos no se consideró un determinado lapso de tiempo; los documentos podían estar escritos en idioma español y en inglés. Para la exclusión de 
los documentos se establecieron los siguientes criterios: investigaciones que no tuvieran en cuenta la población infantil y estudios que explicaran el fenómeno desde otra disciplina diferente a la Psicología. En la Tabla 2 se relaciona el número de documentos hallados en cada una de las bases de datos.

Tabla 2

Número de artículos por base de datos.

\begin{tabular}{lc}
\hline \multicolumn{1}{c}{ Base de datos } & $\begin{array}{c}\text { Número } \\
\text { de artículos }\end{array}$ \\
\hline Dialnet & 6 \\
\hline SciELO & 4 \\
\hline Redalyc & 8 \\
\hline Google Académico & 14 \\
\hline APA PsycNet & 10 \\
\hline PDF Drive & 6 \\
\hline Repositorio Ruidera-UCLM & 1 \\
\hline Repositorio PUCP & 1 \\
\hline
\end{tabular}

Fuente: Elaboración propia.

Dentro de las categorías de análisis se encuentran: acepción de EP y RE; esta categoría tiene como finalidad establecer un panorama de las diversas nociones de los dos conceptos, para de esta manera lograr una comprensión conceptual a partir de las definiciones de distintos autores.

La categoría bidireccionalidad y unidireccionalidad va a permitir reconocer las diferentes direccionalidades que pueden tomar los EP en relación con la RE infantil y el rol de cada uno de los actores dentro del proceso de crianza (cuidadores/niño). El apartado de mutación y variabilidad de los EP, dará luces para establecer la dirección de incidencia de los EP sobre la RE.

\section{Revisión analítica}

\section{Acepción de estilos parentales}

Para comenzar se definirá el concepto de los EP estudiado desde sus inicios por Baumrind (1967), quien refiere que "son comprendidos como una forma manifiesta en que los padres y madres ejercen el proceso de normatización y el manejo de autoridad en sus hijos los cuales fomentan la autorregulación en los mismos" (p. 788); dicha autoridad promueve de manera intencional la autorregulación en los niños. En esta misma línea, Maccoby (1983) adoptan un equivalente del término EP, el cual Ilamaron "estilos de crianza o pautas de crianza", fue descrito como las formas en que los padres o cuidadores se comunican con el niño, el tipo de clima emocional; esto forma una constelación de actitudes acerca del niño, y se puede observar en los comportamientos de los padres o cuidadores.

De esta manera, los estilos de crianza representan la forma de actuar de los padres 0 cuidadores frente a la educación de los niños y por medio de estos se constituyen en los niños hábitos, costumbres y actitudes que van a intervenir en su desarrollo psicoemocional a corto y largo plazo (López y Trujillo, 2012; Esteve, 2005). Por otro lado, Darling y Steinberg (1993) adoptan el concepto de "prácticas educativas" y mencionan que son aquellas habilidades más frecuentes que efectúan los padres frente al comportamiento de sus hijos. En esta misma línea, López y Vesga (2009) estiman que las prácticas educativas empleadas por los padres tienen impacto en el desarrollo emocional de los niños.

Ante las diferentes teorías acerca de los $E P$, en este estudio se decidió profundizar en la teoría propuesta por Diana Baumrind 
(1967, 1975), según Henao y García (2009) es la investigación más reconocida en cuanto a los estilos de educación parental. Baumrind definió los EP como la manifestación de los padres respecto a la forma como llevan a cabo el proceso de normatización y manejo de autoridad sobre los hijos. Según Baumrind los EP se clasifican en tres formas: autoritario, permisivo y autoritativo o democrático (García et al., 2014; Izzedin y Pachajoa, 2009). El estilo autoritario se caracteriza por la existencia de poca comunicación y afecto hacia sus hijos, ejerciendo un alto nivel de control. Los padres permisivos son flexibles, careciendo de control y con aumento en afectividad y comunicación. Mientras que el estilo democrático se distingue por la existencia de límites claros y con argumento, además de la presencia de afectividad y comunicación (Carrillo et al., 2018; Montoya et al., 2016; García et al., 2014; Álvarez, 2010; López y Vesga, 2009; Zarra-Nezhad et al., 2015; Martínez, 2005; Muñoz, 2013; Jorge y González, 2017; Pinta et al., 2019; Aldana y Siena, 2018; Delgado, 2019; Ruiz de Miguel, 1999).

Sin embargo, la teoría de Maccoby (1983) le suma un estilo diferente a los propuestos por Baumrind (1966). Así, estos autores proponen cuatro EP a partir de dos dimensiones: afecto/comunicación y control/establecimiento de límites (como se citó en Capano y Ubach, 2013, p. 87). A partir de estas dimensiones se desarrollan cuatro EP: el autoritario, el permisivo, el democrático y el negligente. Otras investigaciones establecen que los anteriores autores lo que hicieron fue reformular el estilo permisivo, dividiéndolo en dos: el permisivo-indulgente y permisivonegligente. El primero se refiere a padres que muestran una indiferencia frente al tipo de conductas de sus hijos, ya sean positivas o negativas, mostrando pasividad ante ello. El segundo hace relación a padres que no se implican en la crianza de los hijos, mostrando una actitud fría con una suma de maltratos (Losada et al., 2019; Ruiz de Miguel, 1999; Carrillo et al., 2018; Esteve, 2005; Kim y Rohner, 2002; Raya et al., 2009).

Por otra parte, en los años noventa, Darling y Steinberg (1993) plantearon un nuevo modelo en el que existían dos niveles de estudio diferentes respecto a la influencia de los padres sobre los hijos. En el primer nivel se situaría el estilo educativo parental y en el segundo nivel las prácticas parentales con las que se manifestaba ese estilo (Jorge y González, 2017). Por otro lado, estos autores citan a Palacios (1999) quien propone un modelo de construcción conjunta de los EP donde establece que la relación padres e hijos es bidireccional y estos estilos solo toman eficacia si se adecúan a la edad de los niños y promocionan su desarrollo.

De acuerdo con lo mencionado, los EP tienen relación con la RE de los niños; por esta razón, se hace pertinente tener una claridad y comprensión del concepto de regulación emocional. Para ello, en la siguiente categoría se abordará la acepción de RE, la cual abarcará diversas perspectivas teóricas.

\section{Acepción de regulación emocional}

El término regulación emocional es entendido como la capacidad que debe aprender un sujeto para reconocer sus emociones y lograr controlarlas, expresarlas de una manera adecuada y adaptativa frente a situaciones específicas de su vida cotidiana (Piñeres y Siena, 2018). Además, se encontró que el término RE es abordado de diversas maneras, tomando sinónimos para referirse a él tales como: control emocional y autorregulación emocional. Pinta et al. (2019) establecen que el control emocional son las facultades, 
destrezas y habilidades para hacer un uso reflexivo de sus emociones, adoptando un control sobre su sentir. Por su parte, Losada et al. (2019) proponen que la autorregulación emocional es la capacidad de un sujeto de alterar su conducta frente a las exigencias de situaciones específicas. Otros autores definieron la RE como una capacidad que beneficia el funcionamiento del sujeto en su entorno social; afirman que la RE es la capacidad de procesar información sobre los sentimientos propios y ajenos; dicha información se convierte en una guía para que el niño logre un funcionamiento social efectivo al tener control sobre el entorno y sobre conductas y procesos fisiológicos asociados a las emociones (Mayer et al., 2008; Mayer, 1999).

Por otra parte, Goleman $(1995,1996)$ y del Valle (1998) definieron la RE desde una mirada biopsicológica, acuñaron el término de inteligencia emocional, definido como aquella habilidad que tienen los seres humanos para reconocer sus emociones y las de los demás, de motivarse y manejar acertadamente las relaciones interpersonales. Dentro de la inteligencia emocional existe un componente de control emocional que es una habilidad que le permite al ser humano manejar y responder frente a las emociones de forma adecuada, teniendo estrategias para afrontarlas dependiendo de la situación social en la que se encuentre. En esta misma línea, Thompson (1994) y Gross $(1998,1999)$ refieren que la RE consiste en un proceso en el cual las personas intervienen en las emociones teniendo conciencia de ellas, identificando cuándo se experimenta la emoción y cómo se expresa. Años más tarde, Gardner $(1983,2004)$ presenta su teoría de inteligencias múltiples y menciona que existen siete tipos de inteligencia: inteligencia lingüística, inteligencia lógi$\mathrm{ca}$, inteligencia musical, inteligencia visualespacial, inteligencia cinestésica, inteligencia interpersonal e inteligencia intrapersonal. Esta última hace alusión a la $R E$, dado que le permite a la persona comprenderse a sí mismo, sus emociones, sentimientos, para luego tener control sobre ellos. Además, Baumrind (1966) utilizó el término de autorregulación para referirse a "los derechos de un bebé a vivir libremente"; es decir que la RE en un sujeto se logra cuando el mismo es capaz de procesar información sobre sus pensamientos y necesidades (sin que se le obligue a hacerlo) para posteriormente implementar estrategias de autocontrol que le sirvan para implantar sus propias normas de comportamiento.

\section{Estilos parentales}

y regulación emocional

Los EP guardan relación con la RE debido a que, dependiendo del estilo y calidez parental que los padres adopten para llevar a cabo el proceso de normatización en sus hijos, se va a generar el desarrollo de potencialidades afectivas, cognitivas y sociales en los mismos y por ende promoverá en ellos el reconocimiento y regulación de sus emociones tanto positivas como negativas. Así, Baumrind (1966) identifica que las reglas que imponen los EP se logran por medio del uso de la razón (mediados por los reforzadores o castigos); en ese sentido, los EP que ejercen autoridad racional frente a las relaciones complejas de sus hijos van a favorecer en los niños un aprendizaje y modificación de emociones y conductas más aceptables (obediencia) para diferentes situaciones de su vida diaria.

Así, se debe tener en cuenta que el exceso de exigencias y altas demandas por parte de los padres o cuidadores se caracterizan por ser restrictivos y represivos mediante el uso de castigos físicos, lo que dificulta de al guna manera los procesos de RE de sus hijos, lo que los hace rebeldes y agresivos (Baumrind, 1966). 
Es así como se puede evidenciar que la normatización de forma racional (teniendo en cuenta el afecto parental al momento de imponer normas) les permite a los niños la interiorización de respuestas más adaptativas frente a su contexto y estas respuestas suelen ser generadas por medio de la RE, que de alguna manera capacita a los niños para enfrentar situaciones conflictivas (Suárez y Vélez, 2018; Richaud, 2005; Capano y Ubach, 2013). Por el contrario, una normatización irracional le impide al niño muchas veces adquirir conocimiento sobre la manera adecuada de responder ante la interacción con sus pares, o a demandas o conflictos que se le presenten en su diario vivir (Baumrind, 1966).

Sin embargo, ante dicha teoría de Baumrind, diferentes autores han deducido que el EP permisivo indulgente les facilita a los niños lograr una RE, debido a que se caracteriza por la sensibilidad frente a sus necesidades, pudiendo resolver los problemas que se le presenten en la vida cotidiana; lo cual conlleva a la autonomía, la comunicación asertiva, el aprendizaje de sus emociones y por ende una manera adecuada de responder a situaciones específicas de su contexto social (Álvarez, 2010; Pinta et al., 2019; Piñeres y Sienna, 2018; Ruiz de Miguel, 1999; Henao y García, 2009; Zarra-Nezhad et al., 2015; Solís y Manzanares, 2018; Izzedin y Pachajoa, 2009; Varela et al., 2015; Muñoz, 2013).

Así, se considera que el EP autoritativo (democrático) estimula en los niños la implementación de estrategias y destrezas de regulación para sus emociones, ya que el cuidador se interesa mayormente en el acompañamiento, en el apoyo y no en las dificultades de ellos, lo que favorece el desarrollo de dicha RE (Baumrind, 1967; Álvarez, 2010; Pinta et al., 2019; Montoya et al., 2016; Tur, 2014; Piñeres y Sienna, 2018; Ruiz de Miguel,
1999; Henao y García, 2009; Zarra-Nezhad et al., 2015; Solís y Manzanares, 2018; Izzedin y Pachajoa, 2009; Varela et al., 2015). Mientras que el EP autoritario, se considera que al establecer una disciplina estricta y rígida dificulta que los hijos puedan llegar a comprender aquellas normas que inducen a la culpa o amenaza y genera una respuesta condicionada en los niños, la cual los limita a realizar nuevamente una conducta que recibió un castigo, pero también les impide tener un conocimiento sobre las posibles alternativas para dicha conducta. Por ende, este tipo de prácticas le impiden al niño formar estrategias de RE, puesto que limitan la posibilidad de explorar conductas alternativas (Baumrind, 1967).

Unidireccionalidad y bidireccionalidad

Unidireccionalidad y bidireccionalidad son términos a tener en cuenta a la hora de hablar sobre EP, dado que el primer término es utilizado implícitamente dentro de las investigaciones haciendo alusión a que los EP impactan e influyen en el desarrollo emocional (incluida la regulación de emociones), independientemente de las características individuales de los niños; es decir que desde esta perspectiva el niño cumple un rol pasivo. Para dar soporte a lo anterior, las investigaciones muestran que los EP utilizados por los padres o cuidadores para el proceso de normatización y crianza de los niños determinaban el desarrollo emocional y el control que los niños les dieran a sus emociones (Aldana y Siena, 2018; Losada et al., 2019; Henao y García, 2009). Así, Henao y García (2009) citan a Darling y Steinberg (1993), quienes establecen:

Se considera que las prácticas educativas que los padres y madres ponen de manifiesto dentro del núcleo familiar, han de tener impacto 
en el desarrollo de sus hijos e hijas, independiente de las características psicológicas que tenga el niño o la niña. (p. 4)

Por otro lado, en cuanto al tema de bidireccionalidad, en la revisión de antecedentes también se pudo ver que se plantea que los EP y su incidencia en el desarrollo de la regulación de emociones depende no solo de la forma del EP, sino que hay una mediación de los atributos que conforman al individuo. Para lo anterior, Losada et al. (2019) citan a Raznoszczyk et al. (2006), quienes sostienen que "la regulación emocional es fundamental en los primeros años de vida, consideran que el bebé al nacer posee una capacidad de regulación, pero muy frágil y escasa, por ello necesita del ambiente cuidador que le posibilite la regulación" (p. 10). En el proceso bidireccional los niños cumplen un papel activo, donde su desarrollo emocional no solo depende de la forma en que los padres o cuidadores implementan las normas e imponen autoridad para el proceso de crianza, sino que los niños tienen unas características personales que, unidas al acompañamiento que le brinden sus padres, se complementan en el transcurso del desarrollo emocional (Losada et al., 2019; Jorge y González, 2017; Robledo y García, 2008).

\section{Mutabilidad y variabilidad} de los estilos parentales

Teniendo en cuenta que los EP son interpretados como las diferentes formas que los padres o cuidadores adoptan para llevar a cabo el proceso de educación de los niños, estos no son inmutables, dado que ellos se forman, se construyen y cambian dependiendo de las circunstancias, vivencias y desarrollo 0 etapa en que se encuentren los niños. Jorge y González (2017) proponen que los EP se transforman según las circunstancias, tiempo y desarrollo de los hijos. En este mismo orden de ideas, Losada et al. (2019) establecen que: "Ios estilos parentales son generalmente mixtos y van cambiando, ya que varían según el momento evolutivo del niño y con el paso del tiempo" (p. 5). Además, cabe mencionar que la mutabilidad de los EP también se presencia dependiendo del género, etapa evolutiva y características en las que se encuentre el niño (Jorge y González, 2017; Losada et al., 2019; Delgado, 2019; Aldana y Siena, 2018).

Por otro lado, se pudo identificar la variabilidad de los EP, dado que estos se construyen con base en la cultura, valores y creencias, entre otros aspectos que hacen parte de la constitución del ser humano (Izzedin y Pachajoa, 2009; Robledo y García, 2008). Delgado (2019) afirma que: "Los padres a menudo tienen diferentes estilos de crianza en función de la cultura, las características personales y la forma en que se criaron" (p. 9). De igual forma, Aldana y Siena (2018) estipulan que "Ios estilos de socialización parental en cada núcleo familiar son diferentes en su proceso de crianza" (p. 4). Por esta razón, al hablar de los EP se debe tener en cuenta su carácter mutable y variable.

\section{Dificultades y limitaciones}

El establecer un único término para referirse al objeto de estudio fue una dificultad que se presentó a la hora de la revisión documental, dado que, al leer algunos documentos, se evidenció que varios de los autores utilizaban el término estilos parentales indiscriminadamente, ocasionando sesgos a la hora de interpretar y comprender el concepto (Henao y García, 2009; Jorge y González, 2017; Losada et al., 2017). Para ello, hacen uso de las frases "estilos de interacción familiar", "estilos parentales", "prácticas educativas" y "formas de interacción fami- 
liar". Así mismo, Jorge y González (2017) en su artículo se refieren al fenómeno de investigación de dos formas diferentes: "pautas de crianza" y "prácticas de crianza", sin hacer una distinción o aclaración entre ambos conceptos que facilite la comprensión del lector. La anterior dificultad se pudo percibir en la mayoría de artículos revisados, lo cual da cuenta de que los EP deben ser estudiados con más detalle para establecer con claridad el concepto y distinguirlo de otros términos que, aunque hacen parte de la estructuración de los EP, no son sinónimos de ellos. Para dar fundamento a lo anterior, Losada et al. (2019) estipulan que "en la interacción entre distintas variables que se ponen en juego en las prácticas educativas, tales como comunicación, afecto y control entre sus miembros, se van a configurar los distintos estilos educativos" (p. 2). Con esto se puede analizar que las prácticas educativas difieren de los EP, ya que son un componente estructural para los mismos y el término no debe ser usado como semejante a ello.

En cuanto al término regulación emocional también se encontraron diversas formas para referirse a él como: control emocional, regulación emocional, manejo emocional y autorregulación emocional. Estas cuatro formas se utilizaban en los artículos, sin establecer un único término que facilitara la comprensión conceptual del lector. Sin embargo, cabe mencionar que durante la revisión se pudo analizar que las diferentes formas de referirse a la RE proporcionan una similitud conceptual dado que las acepciones van encaminadas a establecer que la RE - 0 las otras formas de nombrar la temática - es la capacidad que tiene el individuo para hacer uso de sus emociones reflexivamente, interviniendo intencionalmente para obtener control sobre las mismas, lo que contribuye a alterar la conducta frente a las exigencias de situaciones específicas
(Pinta et al., 2019; Delgado, 2019; Carillo et al., 2018; Losada et al, 2019; Jorge et al., 2017; Aldana y Siena, 2018; Zarra-Nezhad et al., 2015; Henao y García, 2009).

\section{Conclusiones y recomendaciones}

Durante la revisión bibliográfica se logró comprender que los EP son las diversas formas en las cuales se relacionan los padres con los hijos para el proceso de crianza. Estos resultan esenciales para la RE en los niños, dado que a partir de estas interacciones se proporciona el desarrollo de habilidades afectivas, cognitivas y sociales que a su vez promueven el reconocimiento, control y expresión de emociones (Pinta et al, 2019; Losada et al., 2019; Zarra-Nezhad et al., 2015). Cabe mencionar que, cuando el sujeto logra tener una capacidad de intervenir sobre sus emociones, alterando su comportamiento ante situaciones específicas, esto le facilita la adaptación en su medio-entorno para así responder de forma adaptativa a situaciones estresantes que conlleven a una activación emocional y por ende requiera de un proceso de regulación.

Los EP pueden ser de tipo racional e irracional, siendo los primeros facilitadores para el desarrollo de la RE infantil; dado que, cuando se establecen normas racionales acompañadas de afectividad, teniendo en cuenta el lugar del niño en el proceso de crianza, se contribuye al desarrollo de habilidades de autocontrol que ayudan a la reflexión intencional de las emociones (Zarra-Nezhad et al., 2015).

Por otro lado, los EP irracionales, caracterizados por el establecimiento de normas severas y castigos, pueden llegar a ser una barrera para que el niño desarrolle la capacidad de controlar sus emociones, debido a que inhiben la posibilidad de expresarse, ya que su 
forma irracional de establecer la autoridad en el proceso de crianza restringe la posibilidad de cuestionar y tomar decisiones.

De igual manera, cabe mencionar que diversas investigaciones tienen problemas para establecer un único término para referirse a las temáticas de EP y RE. Esto podría generar sesgos dado que no existiría una claridad conceptual que permita abordar con rigor científico problemáticas sociales que se den a partir de los EP y la RE. Por esta razón, se recomienda para próximas investigaciones hacer una distinción detallada de las diversas formas de referirse a los términos para posibilitar una claridad conceptual en el lector.

A partir de la categoría titulada "Mutación y variabilidad de los estilos parentales" se pudo identificar que los EP cambian en el tiempo y en esto influyen diferentes aspectos como: circunstancias, vivencias y desarrollo 0 etapa en que se encuentren los niños. Lo anterior se debe tener en cuenta en el proceso metodológico de investigaciones aplicadas puesto que es importante reconocer la variabilidad de los EP y el momento evolutivo en el que se encuentra el niño para evitar sesgos en los resultados obtenidos.

Finalmente, se recomienda para próximas investigaciones problematizar acerca de la tendencia de referirse a los EP como formas de interacción que establecen los padres con sus hijos, dado que en Colombia, dentro de las familias, los cuidados de los niños no siempre están bajo la responsabilidad de los padres sino que existen otros parientes que se encargan de este proceso, debido a que la familia en el contexto nacional ha vivenciado cambios a través del tiempo que han modificado su estructura y dinámica. Ante ello, en las estadísticas se ha evidenciado un incremento del $28,7 \%$ de hogares compuestos y extensos, los cuales son conformados por padres e hijos, más parientes 0 amistades; estos últimos en varias ocasiones toman el rol de cuidadores de los menores de edad, debido a las demandas laborales y domésticas que los padres de los niños puedan tener (Departamento Nacional de Planeación [DNP], 2014).

\section{Referencias}

Aldana, M. y Siena, M. (2018). Influencia de los estilos de socialización parental en el desarrollo emocional durante la niñez. [Artículo de revisión, Universidad Autónoma de Bucaramanga]

Álvarez, M. (2010). Prácticas educativas parentales: autoridad familiar, incidencia en el comportamiento agresivo infantil. Revista Virtual Universidad Católica del Norte, 31, 253-273.

Baumrind, D. (1966). Effects of authoritative parental control on child behavior. Child development, 37(4), 887-907.

Baumrind, D. (1967). Child care practices anteceding three patterns of preschool behavior. Genetic Psychology Monographs, 75(1), 43-88. https:// psycnet.apa.org/record/ 1967-05780-001

Baumrind, D. (1975). The contributions of the family to the development of competence in children. Schizophrenia Bulletin, 1(14), 1237. doi:10.1093/SCHBUL/ 1.14.12

Capano, A. y Ubach, A. (2013). Estilos parentales, parentalidad positiva y formación de padres. Ciencias Psicológicas, 7(1), 83-95.

Carrillo, A., Estévez, C. y Gómez, M. (2018). ¿Influyen las prácticas educativas en el desarrollo de la inteligencia emocional de sus hijos? Revista de Psicología, 1(1), 203-212. 
Cuervo, A. (2010). Pautas de crianza y desarrollo socioafectivo en la infancia. Diversitas: Perspectivas en Psicología, 6(1), 111121. https:// www.redalyc.org/articulo. oa? id $=67916261009$

Darling, N. y Steinberg, L. (1993). Parenting Style as Context: An Integrative Model. Psychological Bulletin of American Psychological Association, 113(3), 487-496.

Delgado, D. (2019). Estilos parentales y el desarrollo socioafectivo [Trabajo de grado de pregrado]. Universidad Técnica de Babahoyo.

Departamento Nacional de Planeación (DNP) (2014). Observatorio Nacional de Familias. https://observatoriodefamilia.dnp. gov.co/Documents/Boletines/bolet\% c3\%adn-3---observatorio-de-familias.pdf

Esteve, J. (2005). Estilos parentales, clima familiar $y$ autoestima física en adolescentes [Tesis doctoral]. Universidad de Valencia.

García, J. y Restrepo, J. (2017). Estilos parentales en el proceso de crianza de niños con trastornos disruptivos. Investigación y DesarroIlo, 26(1), 55-74.

García, M., Riveras, S. y Reyes, I. (2014). La percepción de los padres sobre la crianza de los hijos. Acta Colombiana de Psicología, 17(2), 133-141.

Gardner, H. (1983). Frames of mind: the theory of multiples intelligences. Basic Book.

Gardner, H. (2004). The Theory of Multiple Intelligences. Basic Book.

Goleman, D. (1995). Emotional intelligence: Why it can matter more than IQ. Bantam Books.

Goleman, D. (1996). La inteligencia emocional. Kairòs.
Gross, J. J. (1998). Antecedent and Response-Centered Regulation of Emotions: Divergent Implications for Experience, Expression, and Physiology. Journal of Personality and Social Psychology, 74, 224-237.

Gross, J. J. (1999). Emotion regulation: Past, present, future. Cognition \& Emotion, 13, 551-573.

Henao, G. y García, M. (2009). Interacción familiary desarrollo emocional en niños y niñas. Revista Latinoamericana de Ciencias Sociales, Niñez y Juventud, 7(2), 785-802. https:// www. redalyc. org/ articulo. oa?id=77315614009

Izzedin, R. y Pachajoa, A. (2009). Pautas, prácticas y creencias acerca de crianza... ayer y hoy. Revista LIBERABIT, 15(2), 109-115.

Jorge, E. y González, M. C. (2017). Estilos de crianza parental: una revisión teórica. Informes Psicológicos, 17(2), 39-66. http://dx.doi. org/ 10.18566/ infpsic.v17n2a02

Kim, K. y Rohner, R. (2002). Parental warmth, control, and involvement in schooling: Predicting academic achievement among Korean American adolescents. Journal of Cross-Cultural Psychology, 33, 127-140.

Lopez, C. y Trujillo, A. (2012). Estado del arte de la relación entre los estilos parentales y el temperamento en niños y adolescentes [Tesis de maestría]. Universidad de la Sabana.

Losada, A., Estevez, P. y Caronello, T. (2019). Estilos parentales y autorregulación emocional infantil. Revisión narrativa de la literatura. Redes, revista de Psicoterapia Relacional e Intervenciones Sociales, 40, 11-28. https:// dialnet.unirioja.es/servlet/articulo?codigo $=7339240$

Maccoby, E. (1983). Parenting and its effects on children: On Reading and Misreading Behavior Genetics. https:// www.annualreviews. org/ doi/ pdf/ 10.1146/ annurev.psych.51.1.1 
Martínez, I. (2005). Estudio transcultural de los estilos de socialización parental. UCLM.

Mayer, J. (1999). Emotional intelligence. Monographs, 125(2), 209-224.

Mayer, J., Salovey, P. y Caruso, D. (2008). Emotional intelligence. American Psychological Association, 63, 503-517.

Montoya, I., Prado, V., Villanueva, L. y González, R. (2016). Adaptación en la infancia: influencia del estilo parental y del estado de ánimo. Acción Psicológica, 13(2), 15-30. https:// scielo. isciii.es/scielo.php?script=sci_arttext\&pi$d=$ S1578-908X2016000200015

Moreno, J., Espada, J. y Gómez, M. (2020). Role of parenting styles in internalizing, externalizing, and adjustment problems in children. Salud Mental, 43(2), 73-84. http:// www.revistasaludmental.mx/index.php/ salud_mental/article/view/SM.01853325.2020 .011

Muñoz, P. (2013). De la percepción de los estilos parentales a la autonomía [Tesis doctoral]. Universidad Abierta Interamericana.

Pinta, S., Pozo, M., Yepez, E., Cabascango, K. y PiIlajo, A. (2019). Primera infancia: estudio relacional de estilos de crianza y desarrollo de competencias emocionales. CienciAmérica, $8(2), 171-188$.

Piñeres, D. y Siena, M. (2018). Influencia de los estilos de socialización parental en el desarrollo emocional durante la niñez [Trabajo de grado para especialización]. Universidad Autónoma.

Raya, A., Pino, M. J. y Herruzo, J. (2009). La agresividad en la infancia: el estilo de crianza parental como factor relacionado. European Journal of Education and Psychology, 2(2), 211-222.
Richaud, M. (2005). Estilos parentales y estrategias de afrontamiento en niños. Revista Latinoamericana de Psicología, 37(1), 47-58.

Robledo, P. y García, J. (2008). El contexto familiar y su papel en el desarrollo socio-emocional de los niños: revisión de estudios empíricos. Revista de Psicología, 4(1), 75-82. https:// www.redalyc.org/pdf/3498/349832319007. pdf

Ruiz de Miguel, C. (1999). La familia y su implicación en el desarrollo infantil. Revista Complutense de Educación, 10(1), 289. https:// revistas.ucm.es/index.php/RCED/article/ view/RCED9999120289A

Solís, G. y Manzanares, E. (2018). Control psicológico parental y problemas internalizados y externalizados en adolescentes de Lima metropolitana. Revista Colombiana de Psicología, 28(1) 29-47. https://doi.org/10.15446/ rcp.v28n1.66288

Solís, P. y Díaz, M. (2008). Valoración objetiva del estilo de crianza y las expectativas de parejas con niños pequeños. Revista Latinoamericana de Psicología, 40(2), 305-319.

Suárez, P. y Vélez, M. (2018). El papel de la familia en el desarrollo social del niño: una mirada desde la afectividad, la comunicación familiar y estilos de educación parental. Revista Psicoespacios, 12(20): 173- 198.

Thompson, R. (1994). Emotional regulation: a theme in search of definition, the development of emotion regulation. Monographs of the Society for Research in Child Development, 59(25), 25-52.

Tur, A. (2014). Crianza, competencia parental y su relación con el desarrollo de los hijos. Revista Mexicana de Investigación en Psicología, 6(2), 186-191. 
del Valle, A. (1998). Educación de las emociones. Educación, 7(14), 169-198. http://revistas. pucp.edu.pe/index.php/educacion/article/ view/ 5227

Varela, S., Chinchilla, T. y Murad, V. (2015). Prácticas de crianza en niños y niñas menores de seis años en Colombia. Zona Próxima, 22, 193-215. https:// www.redalyc.org/articulo. oa? id $=85339658014$
Zarra-Nezhad, M., Aunola, K., Kiuru, N., Mullola, S. y Moazami-Goodarzi, A. (2015). Parenting Styles and Children's Emotional Development during the First Grade: The Moderating Role of Child Temperament. Journal of Psychology \& Psychotherapy, 5(5). doi: 10.4172/2161-0487.1000206 